\title{
Metformin decreases bone turnover markers in polycystic ovary syndrome: a post hoc study
}

\author{
Shilpa Lingaiah, M.B.B.S., ${ }^{a}$ Laure Morin-Papunen, M.D., Ph.D., ${ }^{a}$ Juha Risteli, M.D., Ph.D., ${ }^{b}$ \\ and Juha S. Tapanainen, M.D., Ph.D. ${ }^{\mathrm{a}, \mathrm{c}}$ \\ ${ }^{a}$ Department of Obstetrics and Gynecology, PEDEGO Research Unit, Medical Research Centre, Oulu University Hospital and \\ University of Oulu, Oulu; ${ }^{b}$ Department of Clinical Chemistry, Oulu University Hospital, Oulu; and ${ }^{\mathrm{c}}$ Department of Obstetrics \\ and Gynecology, University of Helsinki and Helsinki University Hospital, Helsinki, Finland
}

Objective: To study the effects of metformin treatment on bone turnover in women with polycystic ovary syndrome (PCOS), as measured by serum concentrations of bone turnover markers.

Design: Post hoc study of a previously conducted prospective multicenter, placebo-controlled, randomized study.

Setting: University clinic.

Patient(s): The study cohort consisted of 74 non-obese women (body mass index $<27 \mathrm{~kg} / \mathrm{m}^{2}$ ) and 44 obese women (body mass index $\geq 27 \mathrm{~kg} / \mathrm{m}^{2}$ ) diagnosed with PCOS, with a mean age of $27.6 \pm 4.0$ (SD) years.

Intervention(s): Randomization to receive metformin or placebo for 3 months.

Main Outcome Measure(s): Serum levels of bone formation marker procollagen type I amino-terminal propeptide (PINP) and bone resorption marker carboxy-terminal cross-linking telopeptide of type I collagen (CTX) at baseline and after metformin/placebo treatment.

Result(s): Serum levels of PINP and CTX were similar between the metformin and placebo groups at baseline in the whole study population. Obese women, when compared with non-obese, had lower baseline levels of PINP and CTX. Levels of PINP and CTX were significantly reduced in the whole study population, as well as in both non-obese and obese women after 3 months of metformin treatment, whereas no significant changes were observed in the placebo group.

Conclusion(s): Metformin treatment, when compared with placebo, was associated with reduced bone turnover, as suggested by reductions in markers of bone formation and resorption, leading to slower bone remodeling in premenopausal women with PCOS.

Clinical Trial Registration Number: NCT00994812. (Fertil Steril ${ }^{\circledR} 2019 ; \mathbf{\square}$ - $\mathbf{0}$. C2019 by American Society for Reproductive Medicine.)

Key Words: Bone turnover markers, carboxy-terminal cross-linking telopeptide of type I collagen (CTX), metformin, polycystic ovary syndrome, procollagen type I amino-terminal propeptide (PINP)

Discuss: You can discuss this article with its authors and other readers at https://www.fertstertdialog.com/users/16110-fertilityand-sterility/posts/47362-27473

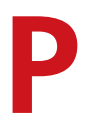

olycystic ovary syndrome (PCOS) is a common endocrine disorder in women of reproductive age, with a prevalence of $6 \%-$ $15 \%$, depending on the diagnostic criteria used (1). Women with PCOS show heterogeneity of characteristics, including oligo/amenorrhea, hyperandrogenism, obesity, insulin resistance, and hyperinsulinemia. Peak skeletal mass is attained from late adolescence to the early thirties, and menstrual dysfunction during this period might influence the bone mass accrued.

Received December 13, 2018; revised March 31, 2019; accepted April 5, 2019.

S.L. has nothing to disclose. L.M.-P. has nothing to disclose. J.R. has nothing to disclose. J.S.T. has nothing to disclose.

Supported by grants from the Sigrid Jusélius Foundation, Finland, the Academy of Finland, and the Medical Research Centre Oulu, Finland, Oulu University Hospital, Finland, and University of Oulu, Finland.

Reprint requests: Juha S. Tapanainen, M.D., Ph.D., University of Helsinki and Helsinki University Central Hospital, Department of Obstetrics and Gynecology, P.O. Box 140, 00029 HUS, Finland. (E-mail: juha.tapanainen@helsinki.fi).

Fertility and Sterility® Vol. $\mathbf{\square}$, No. $\mathbf{\square}, \mathbf{\square} 2019$ 0015-0282/\$36.00

Copyright (02019 Published by Elsevier Inc. on behalf of the American Society for Reproductive Medicine

https://doi.org/10.1016/j.fertnstert.2019.04.013

Furthermore, both androgens and estrogens have an independent and possibly additive association with peak bone mass attainment and maintenance (2). It has been postulated that hormonal imbalance in women with PCOS might have a negative effect on bone formation and bone mineral density (BMD) (3), but whether it predisposes to osteoporosis in later life remains elusive. Furthermore, few studies have even reported lower BMD in women with PCOS compared with their healthy counterparts $(4,5)$.

Metformin is one of the widely used drugs for the treatment of PCOS and acts by inhibiting hepatic glucose production and increasing peripheral 
tissue sensitivity to insulin. Long-term treatment with metformin has been shown to normalize ovulation, menstrual cyclicity, and hyperandrogenism (6). Although the exact mechanism is not fully understood, it is thought that metformin lowers glucose production via activation of the $5^{\prime}$ adenosine monophosphate-activated protein kinase (AMPK) pathway (7). Further, AMPK subunits are highly expressed in bone tissue, osteoblasts, and osteoclasts. Cellular and animal studies have reported that metformin has a direct osteogenic effect and bone loss inhibiting effect (8). Few clinical studies have evaluated the effects of metformin on bone metabolism and bone turnover in diabetics, suggesting a beneficial effect on bone (9). However, there are only limited data as regards its effect on bone metabolism and measures of bone turnover in women with PCOS.

Bone, being a metabolically active tissue, undergoes continuous remodeling, wherein bone formation by osteoblasts is coupled to bone resorption by osteoclasts. Bone formation and resorption can be determined indirectly by the measurement of serum concentrations of various biomarkers (i.e., bone matrix components released into the circulation during bone formation or resorption). The serum concentrations of bone turnover markers (BTMs) reflect bone remodeling and can be used as markers of the rate of bone formation and resorption. These markers allow noninvasive assessment of bone turnover and are sensitive enough to reflect acute changes in it, providing a more representative view of overall bone loss than that obtained by measuring the rates of change in BMD at specific skeletal sites (10).

Ninety percent of the bone matrix is composed of type I collagen, which is synthesized as a precursor procollagen, cleavage of which releases procollagen type I aminoterminal propeptide (PINP) into the circulation. The carboxy-terminal cross-linking telopeptide of type I collagen (CTX) is released from the bone matrix during resorption and reflects the degradation of type I collagen. Thus, PINP and CTX reflect the rates of bone formation and resorption, respectively (11). The International Osteoporosis Foundation and the International Federation of Clinical Chemistry and Laboratory Medicine have recommended the use of serum PINP and CTX as reference biochemical markers of bone formation and resorption, respectively (12).

The present study was a post hoc secondary study among a subset of patients who have been described in a previously published prospective multicenter, placebocontrolled, randomized study on the effects of metformin on miscarriage, pregnancy and live-birth rates, which showed that metformin treatment compared with placebo improved pregnancy and live-birth rates in women with PCOS (13). The aim of the present study was to investigate the effects of metformin on bone turnover markers in women with PCOS. In line with the recommendations of the International Osteoporosis Foundation and the International Federation of Clinical Chemistry and Laboratory Medicine, PINP and CTX were used as reference biochemical markers of bone formation and resorption.

\section{MATERIALS AND METHODS}

\section{Subjects}

The study population consisted of 118 Caucasian women (mean age $27.6 \pm 4.0$ [SD] years, mean body mass index [BMI] $26.5 \pm 6.0 \mathrm{~kg} / \mathrm{m}^{2}$ ) diagnosed with PCOS according to the European Society of Human Reproduction and Embryology/American Society for Reproductive Medicine (ESHRE/ ASRM) consensus definition (14). The present study was a post hoc analysis among a subset of subjects who were selected from a large cohort of subjects in a prospective multicenter, placebo-controlled randomized study on the effects of metformin on miscarriage, pregnancy, and live-birth rates (13). Only the subjects who were examined at Oulu University Hospital were included in the present study. The primary study was registered under the clinical trial registration number NCT00994812 and was approved by the Ethics Committee of the Northern Ostrobothnia Hospital District (1396/2004) and the National Supervisory Authority for Welfare and Health (D1339/05.01.00.06/2009). The primary study was conducted during 2003-2009, with the first patient enrolment on November 10, 2002.

Women who became pregnant or had a miscarriage before the study period of 3 months were excluded from the present study. All women in the present study were examined and recruited at Oulu University Hospital during 2003-2009. Informed written consent was obtained from all the subjects. Women with diabetes, active liver disease (alanine aminotransferase $>100 \mathrm{IU} / \mathrm{L}$ ), past or present cardiac failure (New York Heart Association I-IV), and liver or renal failure (s-creatinine $>124 \mu \mathrm{mol} / \mathrm{L}$ ), women who used alcohol and hormone preparations, smokers, and pregnant and lactating women were excluded from the original study. None of the study subjects were using medications known to affect hormonal or metabolic parameters or bone metabolism, and none had a history of fracture in the preceding 6 months. The subjects were not allowed to use calcium, vitamin D, dietary supplements, herbal therapies, or vitamins during the study.

Non-obese women (BMI $<27 \mathrm{~kg} / \mathrm{m}^{2}$ ) received metformin (Diformin; Leiras) at a dose of $500 \mathrm{mg}+1,000 \mathrm{mg}$ daily, or placebo; obese women (BMI $\geq 27 \mathrm{~kg} / \mathrm{m}^{2}$ ) received metformin at a dose of 1,000 mg twice daily, or placebo. The limit for BMI was chosen on the basis of earlier studies that indicated increased insulin resistance in women with PCOS at a BMI of $27 \mathrm{~kg} / \mathrm{m}^{2}$ (15). The dose of $1,500 \mathrm{mg}$ of metformin for non-obese women with PCOS and 2,000 $\mathrm{mg}$ for obese women was based on earlier studies (16-18), which showed that $1,500 \mathrm{mg}$ and 2,000 $\mathrm{mg}$ of metformin was effective enough to restore ovulation in most of the non-obese and obese women with PCOS, respectively, and to improve hyperandrogenism and insulin sensitivity significantly. Furthermore, using a smaller dose in non-obese women was to minimize possible side effects, and thereby dropouts.

Clinical, metabolic, and hormonal parameters were evaluated 1-7 days after spontaneous menstruation in oligomenorrheic subjects or at any other convenient time in amenorrhoeic subjects. A second evaluation was scheduled 


\section{TABLE 1}

Characteristics of the study population.

$\begin{array}{lccc}\text { Characteristic } & \begin{array}{c}\text { Metformin } \\ (\mathbf{n}=\mathbf{5 7})\end{array} & \begin{array}{c}\text { Placebo } \\ (\mathbf{n}=\mathbf{6 1 )}\end{array} & \text { All subjects } \\ \text { PCO + OA + HA } & 21(36.8) & 16(26.2) & 37(31.4) \\ \text { PCO + OA } & 35(61.4) & 44(72.1) & 79(66.9) \\ \text { PCO + HA } & 1(1.8) & 1(1.6) & 2(1.7)\end{array}$

Note. Values are number (percentage). $\mathrm{HA}=$ hyperandrogenism (serum $\mathrm{T}$ level $>2.3 \mathrm{nmol} / \mathrm{L}$ and/or Ferriman-Gallwey hirsutism score of $>7$ ); $\mathrm{OA}=$ oligoamenorrhea; $\mathrm{PCO}=$ polycystic ovaries on ultrasonography.

Lingaiah. Metformin lowers BTMs in PCOS. Fertil Steril 2019.

3 months after the first visit. Fifty-seven women received metformin and 61 received placebo for 3 months. Blood samples were collected in a fasting state at baseline and at 3 months of treatment with metformin/placebo and were stored at $-20{ }^{\circ} \mathrm{C}$ until the time of analysis.

All study subjects had polycystic ovaries in ultrasonography according to the ESHRE/ASRM definition (14); the majority of them had oligoamenorrhea ( $n=116,98.3 \%$ ), and 39 (33.1\%) displayed hyperandrogenism (serum $\mathrm{T}$ level $>2.3 \mathrm{nmol} / \mathrm{L}$, according to the upper limits of our accredited laboratory in fertile-aged women and/or Ferriman-Gallwey hirsutism score of $>7$ ) (Table 1). Other patient characteristics at baseline and after 3 months of treatment are shown in Table 2 .

\section{Assays}

Serum concentrations of PINP, CTX, and 25-hydroxyvitamin D (250HD) were determined using the IDS-iSYS Multi-Discipline Analyser (Immunodiagnostics Systems) based on chemiluminescence technology, according to the manufacturer's protocol. In brief, the samples were incubated with specific antibodies, followed by the addition of streptavidin-coated magnetic particles. The magnetic particles were captured, and trigger reagents were added after further incubation. The concentration of analytes in the original sample was directly proportional to the resulting light emitted by the acridinium label. The reportable ranges of the assays for PINP, CTX, and 250HD were 2-230 $\mu \mathrm{g} / \mathrm{L}, 0.033-6 \mu \mathrm{g} / \mathrm{L}$, and 5-140 $\mu \mathrm{g} / \mathrm{L}$, respectively. The respective intra- and interassay coefficients of variation were $4 \%$ and $2.2 \%$ for PINP, $2.3 \%$ and 1.8\% for CTX, and 5.1\% and 13\% for 250HD.

Serum concentrations of sex hormone-binding globulin (SHBG), androstenedione (A), dehydroepiandrosterone sulfate (DHEAS), and $\mathrm{E}_{2}$ were analyzed, and oral glucose tolerance tests were carried out after an overnight fast as described earlier (13). Serum T was analyzed using Agilent triplequadrupole 6410 liquid chromatography/mass spectrometry equipment with an electrospray ionization source operating in positive-ion mode (Agilent Technologies) as detailed earlier (13). Multiple reaction monitoring was used to quantify $\mathrm{T}$ by trideuterated T. Intra-assay coefficients of variation of the method were $5.3 \%, 1.6 \%$, and $1.2 \%$ for $\mathrm{T}$ at $0.6,6.6$, and $27.7 \mathrm{nmol} / \mathrm{L}$, respectively. Interassay coefficients of variation were $5.3 \%, 4.2 \%$, and $1.0 \%$ for the respective concentrations. The free androgen index (FAI) was calculated using the equa- tion: $100 \times \mathrm{T} / \mathrm{SHBG}$ (both as nmol/L). Homeostatic model assessment of insulin resistance (HOMA-IR) and the wholebody insulin sensitivity index (i.e., the Matsuda index) were calculated to quantify the degree of insulin resistance $(19,20)$.

\section{Statistical Methods}

Statistical analyses were performed using SPSS 25.0 software (IBM). Variables with skewed distribution underwent logarithmic transformation. Independent samples $t$ tests were used for comparisons between the metformin and placebo groups, and paired-samples $t$ tests were used to evaluate changes between the measurements at baseline and after 3 months of treatment within the groups. General linear modeling was used to evaluate the significant determinant of changes in the levels of BTMs. Analysis of correlation between parameters was performed by using Pearson's correlation coefficient. A value of $P<.05$ was considered statistically significant.

\section{RESULTS}

\section{Baseline Comparisons and Changes after 3 Months of Metformin/Placebo Treatment in Non-obese and Obese Women}

Clinical, hormonal, and metabolic parameters were comparable between metformin and placebo groups at baseline in the non-obese and obese women (Table 2). There was a small but statistically significant decrease in weight $(P=.043)$ and BMI $(P=.049)$ in the obese group after metformin treatment. In addition, the concentrations of $\mathrm{T}(P=.014)$ and fasting glucose $(P=.004)$ significantly decreased, and the Matsuda index significantly increased $(P=.046)$. In the non-obese group treated with metformin, the concentrations of $\mathrm{T}$ $(P=.001)$, the FAI $(P<.001)$, and A $(P=.001)$ significantly decreased. No statistically significant changes were observed in any of the clinical, hormonal, and metabolic parameters during placebo treatment in the non-obese and obese groups.

\section{Baseline Comparisons of BTMs and 250HD}

The baseline levels of PINP $(P=.307)$, CTX $(P=.980)$, and 250HD $(P=.281)$ did not differ between the metformin and placebo groups in the whole study population. However, obese women when compared with non-obese women had significantly lower levels of PINP (39.6 \pm 15.9 [mean \pm SD] $\mu \mathrm{g} / \mathrm{L}$ vs. $50.0 \pm 21.6 \mu \mathrm{g} / \mathrm{L}, P=.003)$ and CTX $(0.32 \pm$ $0.14 \mu \mathrm{g} / \mathrm{L}$ vs. $0.46 \pm 0.21 \mu \mathrm{g} / \mathrm{L}, P<.001)$, and similar levels of $250 \mathrm{HD}(22.0 \pm 8.0 \mu \mathrm{g} / \mathrm{L}$ vs.19.5 $\pm 6.4 \mu \mathrm{g} / \mathrm{L}, P=.076)$. Furthermore, in both metformin and placebo groups, obese women had lower levels of PINP and CTX compared with non-obese women, though the difference in PINP levels did not reach statistical significance in the metformin group (Table 3).

\section{Changes in BTMs and 250HD after 3 Months of Metformin/Placebo Treatment}

The levels of PINP and CTX were significantly decreased after 3 months of metformin treatment in both non-obese and 


\section{TABLE 2}

Clinical, hormonal, and metabolic parameters at baseline and after 3 months of treatment with metformin/placebo in the study population.

\begin{tabular}{|c|c|c|c|c|c|c|c|c|}
\hline \multirow[b]{3}{*}{ Parameter } & \multicolumn{4}{|c|}{ Non-obese $\left(B M I<27 \mathrm{~kg} / \mathrm{m}^{2}\right)(\mathrm{n}=74)$} & \multicolumn{4}{|c|}{ Obese $\left(B M I \geq 27 \mathrm{~kg} / \mathrm{m}^{2}\right)(n=44)$} \\
\hline & \multicolumn{2}{|c|}{ Metformin $(n=40)$} & \multicolumn{2}{|c|}{ Placebo $(n=34)$} & \multicolumn{2}{|c|}{ Metformin $(n=17)$} & \multicolumn{2}{|c|}{ Placebo $(n=27)$} \\
\hline & Baseline & $3 \mathrm{mo}$ & Baseline & $3 \mathrm{mo}$ & Baseline & $3 \mathrm{mo}$ & Baseline & $3 \mathrm{mo}$ \\
\hline Age (y) & $27.1(3.1)$ & & $27.9(4.2)$ & & $28.8(3.8)$ & & $27.3(5.0)$ & \\
\hline Weight (kg) & $61.0(7.8)$ & $60.4(7.5)$ & $62.3(8.7)$ & $62.3(8.7)$ & 89.7 (11.7) & $88.4(11.8)^{\mathrm{a}}$ & $90.0(14.1)$ & $90.1(14.0)$ \\
\hline BMI $\left(\mathrm{kg} / \mathrm{m}^{2}\right)$ & $22.5(2.2)$ & $22.3(2.2)$ & $22.7(2.6)$ & $22.7(2.5)$ & $33.4(4.3)$ & $32.9(4.4)^{\mathrm{b}}$ & $33.3(4.4)$ & $33.3(4.5)$ \\
\hline WHR & $0.76(0.05)$ & $0.76(0.06)$ & $0.78(0.06)$ & $0.78(0.07)$ & $0.83(0.06)$ & $0.83(0.05)$ & $0.85(0.05)$ & $0.84(0.05)$ \\
\hline Hirsutism score & $4.8(3.1)$ & $5.1(3.3)$ & $4.9(3.2)$ & $4.6(2.8)$ & $7.3(3.7)$ & $7.0(3.9)$ & $6.7(4.9)$ & $6.0(4.7)$ \\
\hline $\mathrm{E}_{2}(\mathrm{pmol} / \mathrm{L})$ & 209.9 (93.8) & $204.9(158.8)$ & $231.2(98.4)$ & 236.5 (133.4) & 204.3 (53.6) & 207.2 (98.1) & $197.4(108.4)$ & $200.2(95.1)$ \\
\hline$(\mathrm{nmol} / \mathrm{L})$ & $1.6(0.7)$ & $1.2(0.6)^{c}$ & $1.7(0.7)$ & $1.6(0.6)$ & $1.6(0.7)$ & $1.3(0.5)^{d}$ & $1.4(0.6)$ & $1.5(1.0)$ \\
\hline SHBG (nmol/L) & $59.0(20.2)$ & $70.0(41.3)$ & $56.6(18.1)$ & $60.9(27.0)$ & $43.1(13.7)$ & $41.9(16.1)$ & $35.1(13.7)$ & 36.6 (30.4) \\
\hline & $3.0(1.9)$ & $2.1(1.3)^{c}$ & $3.3(2.2)$ & $3.1(1.9)$ & $4.2(2.1)$ & $3.6(2.1)$ & $4.8(3.5)$ & $5.0(3.4)$ \\
\hline HEAS ( $\mu \mathrm{mol} / \mathrm{L})$ & $5.3(2.3)$ & $5.6(2.5)$ & $6.2(3.3)$ & $6.0(2.7)$ & $4.9(2.1)$ & $5.3(2.2)$ & $5.4(2.3)$ & $5.3(1.9)$ \\
\hline $\mathrm{A}(\mathrm{nmol} / \mathrm{L})$ & $17.8(9.5)$ & $14.6(5.7)^{\mathrm{e}}$ & $21.4(7.7)$ & $20.0(8.1)$ & $15.7(5.4)$ & $14.5(5.7)$ & 17.1 (6.7) & $17.9(8.0)$ \\
\hline $\begin{array}{l}\text { Fasting glucose } \\
\text { (mmol/L) }\end{array}$ & $5.0(0.5)$ & $4.9(0.4)$ & $5.1(0.4)$ & $5.0(0.4)$ & $5.3(0.4)$ & $5.1(0.3)^{\dagger}$ & $5.3(0.3)$ & $5.3(0.3)$ \\
\hline $\begin{array}{l}\text { Fasting insulin } \\
(\mathrm{mU} / \mathrm{L})\end{array}$ & $5.6(3.1)$ & $5.8(2.8)$ & $6.4(2.8)$ & $7.7(6.5)$ & $17.2(17.0)$ & $12.1(5.9)$ & $14.5(6.5)$ & $15.0(7.9)$ \\
\hline HOMA-IR & $1.3(0.7)$ & $1.3(0.6)$ & $1.5(0.7)$ & $1.8(1.7)$ & $4.2(3.8)$ & $2.8(1.4)$ & $3.4(1.6)$ & $3.6(1.9)$ \\
\hline Matsuda index & $8.3(3.7)$ & $9.0(4.7)$ & $7.3(3.6)$ & $6.6(3.4)$ & $3.5(2.3)$ & $4.2(2.0)^{9}$ & $3.4(2.1)$ & $3.7(3.3)$ \\
\hline
\end{tabular}

obese women, whereas no significant differences were observed in the placebo group (Fig. 1, Table 3). The average declines from the baseline values were $25.7 \%$ for PINP and $31.1 \%$ for CTX in the non-obese women $(P<.001$ for both) and $32 \%$ for PINP $(P<.001)$ and $24.1 \%$ for CTX $(P=.022)$ in the obese women. Concentrations of 250HD increased in both non-obese and obese women in the metformin and placebo groups, although statistically significant differences were observed only in non-obese women in the metformin group and obese women in the placebo group.

\section{Baseline Comparisons of BTMs and 25OHD, and Changes after 3 Months of Metformin/Placebo Treatment in Normoandrogenic and Hyperandrogenic Women}

The subjects were further divided into normoandrogenic (NA) and hyperandrogenic (HA) (serum T level $>2.3 \mathrm{nmol} / \mathrm{L}$ and/or Ferriman-Gallwey hirsutism score of $>7$ ). The baseline concentrations of PINP, CTX, and 250HD were comparable between NA and HA women in the metformin and placebo groups. The levels of PINP and CTX were significantly decreased after 3 months of metformin treatment in both NA $(P<.001$ for PINP and CTX $)$ and HA women $(P=.002$ for PINP, $P=.001$ for CTX), whereas no significant differences were observed in the placebo group (Supplemental Table 1, available online). The average declines from the baseline values were 30\% for PINP and 31\% for CTX in NA women, and $22.8 \%$ for PINP and 27\% for CTX in HA women. The levels of 250HD were increased in NA women in both metfor$\min (P=.013)$ and placebo groups $(P=.006)$.

\section{Correlation Analyses and General Linear Modeling}

Changes in PINP and CTX levels did not show any statistically significant correlations when compared with changes in the levels of $E_{2}$, T, SHBG, the FAI, A, DHEAS, fasting glucose, fasting insulin, HOMA-IR, or Matsuda index during metformin treatment. In general linear modeling, only metformin treatment, not BMI group or androgenic status, showed a statistically significant interaction with the changes in the levels of PINP $(P<.001)$ and CTX $(P=.001)$. As regards the changes in 250HD levels, metformin treatment $(P=.772)$ and BMI $(P=.442)$ did not show any significant interactions.

\section{DISCUSSION}

The present study showed that serum levels of the bone formation marker PINP and the bone resorption marker CTX significantly decreased during treatment with metformin in women with PCOS compared with those treated with placebo. During 3 months of metformin treatment, the average declines of PINP and CTX levels from baseline values were $27.4 \%$ and $30 \%$, respectively, in the whole population. Furthermore, the significant decreases in the levels of PINP and CTX were observed in both non-obese and obese women with PCOS in the metformin group.

Bone turnover depends on bone formation and resorption through cross-talk between osteoblasts and osteoclasts. Reduced levels of markers of bone formation and resorption are associated with low bone turnover and a slower rate of bone loss. Studies have shown that low bone turnover could slow bone loss and give rise to a bone density exceeding that expected for age. Conversely, increased bone turnover 


\section{TABLE 3}

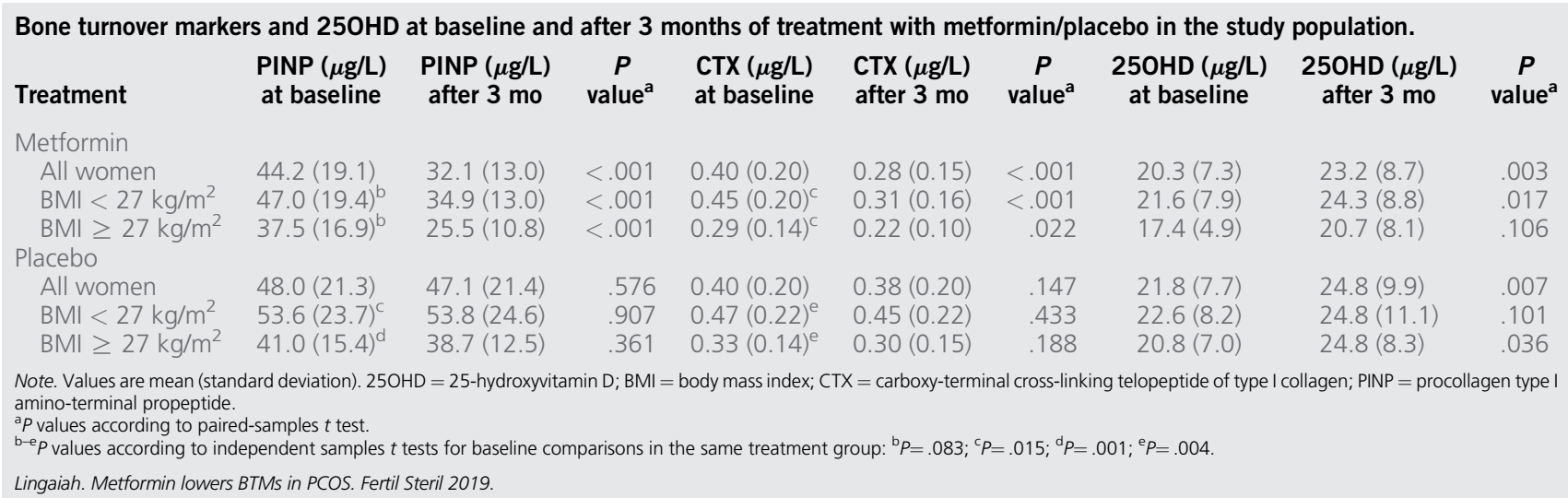

is associated with accelerated bone loss and potential deterioration in bone quality $(8,21)$. Bone turnover markers reflect whole-body bone turnover, underlying changes in bone mass and bone histomorphometric parameters, and are thus predictive of total-body bone loss. Furthermore, there is a moderate association between baseline levels of BTMs and subsequent changes in BMD (22).

Cellular studies have shown that metformin is a potent stimulator of AMPK activation in osteoblasts, resulting in their differentiation and mineralization, and stimulates type 1 collagen production in osteoblast-like cell lines, suggesting a direct osteogenic effect (23-25), whereas few studies have not shown such an effect $(26,27)$. It has been reported that treatment with metformin prevents bone loss in ovariectomized rats, suggesting protective effects of metformin against bone loss $(28,29)$. In contrast, one study showed that metformin has no effect on bone mass in rodents (30).

\section{FIGURE 1}
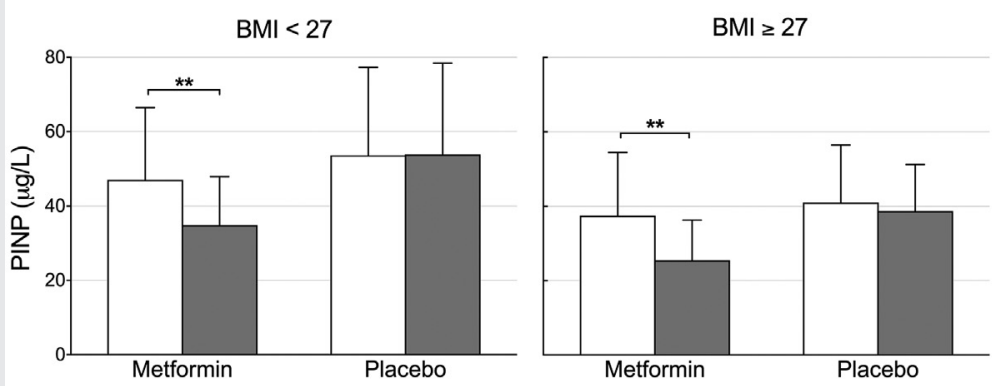

Baseline

3 months
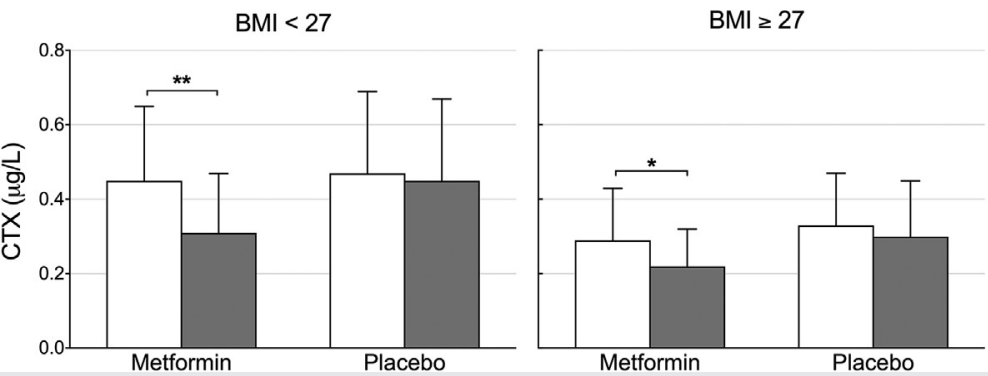

Concentrations of bone turnover markers at baseline and after 3 months of metformin/placebo treatment in women with polycystic ovary syndrome. The bars represent means and the error bars standard deviations. CTX $=$ carboxy-terminal cross-linking telopeptide of type I collagen; PINP $=$ procollagen type I amino-terminal propeptide. ${ }^{*} P=.022 ; * *<.001$.

Lingaiah. Metformin lowers BTMs in PCOS. Fertil Steril 2019. 
Studies on the effects of metformin on bone turnover in PCOS are still lacking, even though metformin is widely used in the treatment of the condition. In clinical studies, the effect of metformin on bone has been investigated mainly in diabetics. It has been reported that metformin reduces fracture risk in patients with type 2 diabetes mellitus (T2DM) (9), whereas one study found no association between metformin and fracture incidents (31). Furthermore, it has also been reported that metformin decreases the markers of bone formation and resorption and bone remodeling in T2DM (32).

In the present study, the baseline levels of BTMs in obese women with PCOS were already decreased when compared with non-obese women. Similar results have been observed in healthy premenopausal women with higher BMI (33). It has been postulated that higher BMI may be associated with increased secretion of various hormones from adipocytes (including estrogen), influencing osteoblast and osteoclast activity (34). In the present study, however, the $E_{2}$ levels in obese women with PCOS were not increased when compared with those of non-obese women. Furthermore, the concentrations of $E_{2}$ remained unchanged throughout the treatment period in both groups, suggesting that the decrease in BTMs may not be related to $E_{2}$ effect.

Androgens have been shown to influence bone metabolism directly through their action on osteoblasts by promoting bone formation, and also indirectly by inhibition of bone resorption (2). Furthermore, SHBG plays a crucial role in bone metabolism and remodeling because it binds to $\mathrm{T}$ and $17 \beta-\mathrm{E}_{2}$, thereby regulating their bioavailability and access to target cells (35), and may play a role in the determination of bone mass in premenopausal women. In the present study, the concentrations of $\mathrm{T}$ decreased in both non-obese and obese women with PCOS, and FAI decreased in nonobese women treated with metformin, which might be associated with decreased levels of BTMs. However, the changes in $\mathrm{T}$, SHBG, and FAI levels during metformin treatment did not correlate with the changes in the levels of BTMs. Furthermore, the decrease in the levels of BTMs was not dependent on the androgen status of the women, because both NA and HA women with PCOS showed similar declines.

Increased mechanical loading secondary to increased body weight stimulates bone formation through stimulation of osteoblast activity (36). Body weight, covering fat mass and lean mass, has an impact on both bone turnover and bone density. In the present study, there was a decrease in BTMs in both obese and non-obese subjects in the metformin group, suggesting that metformin, not body weight, was the influencing factor in bone turnover. It is possible that the effect of metformin on BTMs is mediated via other mechanisms at the cellular level, which has to be investigated in future studies.

The important prohormone 250HD influences BMD by regulating calcium metabolism, but 250HD per se may not have any significant influence on BTMs (37). Even though 250HD levels showed an increasing trend in non-obese and obese subjects in both treatment groups, significant increases were observed only in the non-obese subjects treated with metformin and obese subjects treated with placebo. It must be noted that the seasonal variation of 250HD levels was not taken into account in the present study, which could be one explanation for the differences observed between the two treatment groups. Furthermore, studies on the effect of metformin on 250HD levels are sparse, with conflicting results. It has been reported that treatment with metformin in T2DM has no effect on 250HD levels $(38,39)$, whereas one study revealed that metformin improves 250HD levels (40).

According to the Endocrine Society Clinical Practice Guideline (41), almost half (51\%) of our study population was vitamin D deficient [25(OH)D below $20 \mu \mathrm{g} / \mathrm{L}]$, and 34\% had vitamin D insufficiency [25(OH)D of 21-29 $\mu \mathrm{g} / \mathrm{L}$ ]. This is in line with studies reporting low levels of 250HD in women with PCOS (42). Bone turnover markers decreased significantly in the metformin group compared with the placebo group in women with both deficient and insufficient vitamin D levels (Supplemental Table 2), suggesting that the decrease in BTMs was not dependent on vitamin D levels in our study population.

There are several strengths as well as limitations in our study. A potential limitation may be the selection of the study subjects: this is a post hoc analysis of a previously conducted study. However, the subjects who participated in the present study did not differ from the subjects of the primary study as regards PCOS phenotypes or anthropometric, hormonal, and metabolic parameters (data not shown). The duration of the treatment was 3 months, which may be a limiting factor. There is an interplay between resorption and formation locally in bone, meaning that when resorption increases formation increases, and vice versa. However, resorption is a faster process (2 to 3 weeks) when compared with formation (3 months) $(43,44)$. Thus, the present study period of 3 months should have been sufficient to depict changes in bone formation and resorption reflected by BTMs. The factors leading to biological variability in BTMs were minimized, because all blood samples were collected in a fasting state. This is particularly important as regards CTX levels, because they decrease by approximately 20\% after food intake (11). Because none of the study subjects had any active liver disease or history of renal failure, the effects of clearance of PINP and CTX from the circulation by hepatic endothelium and the kidneys were controlled. The timing of samples was not scheduled according to the season, but earlier studies have shown that there is no significant seasonal variation in the levels of BTMs (45). The samples were taken during the follicular phase in oligomenorrheic women and at any time in amenorrhoeic women. Previous studies have shown that variations in the levels of BTMs over the menstrual cycle are so small that the effect of the menstrual cycle can be considered to be insignificant $(10,11)$. The effect of oligo-amenorrhea compared with regular menstrual cycles on BTMs could not be analyzed in the present study, because there were few women with regular cycles. Even though the study period of 3 months was sufficient to show the changes in the levels 
of BTMs, the effect of treatment with metformin should be assessed during a longer study period to account for its effect on various hormonal and metabolic parameters.

In conclusion, metformin treatment of premenopausal women with PCOS for 3 months was associated with reduced bone turnover, as suggested by reductions in markers of bone formation and resorption, leading to slower bone remodeling preventing bone loss. However, long-term intervention studies with BMD measurements and fracture assessment are necessary to demonstrate the effects of metformin on bone turnover and remodeling in PCOS conclusively.

Acknowledgments: The authors thank Katja Koukkula for skillful technical assistance; Risto Bloigu for statistical advice; and Nick Bolton for language revision.

\section{REFERENCES}

1. March WA, Moore VM, Willson KJ, Phillips DI, Norman RJ, Davies MJ. The prevalence of polycystic ovary syndrome in a community sample assessed under contrasting diagnostic criteria. Hum Reprod 2010;25:544-51.

2. Zborowski JV, Cauley JA, Talbott EO, Guzick DS, Winters SJ. Bone minera density, androgens, and the polycystic ovary: the complex and controversial issue of androgenic influence in female bone. J Clin Endocrinol Metab 2000; 85:3496-506.

3. Krishnan A, Muthusami S. Hormonal alterations in PCOS and its influence on bone metabolism. J Endocrinol 2017;232:R99-113.

4. Kirchengast S, Huber J. Body composition characteristics and body fat distribution in lean women with polycystic ovary syndrome. Hum Reprod 2001;16:1255-60.

5. Yüksel O, Dökmetaş HS, Topcu S, Erselcan T, Şencan M. Relationship between bone mineral density and insulin resistance in polycystic ovary syndrome. J Bone Miner Metab 2001;19:257-62.

6. Diamanti-Kandarakis E, Christakou CD, Kandaraki E, Economou FN Metformin: an old medication of new fashion: evolving new molecular mechanisms and clinical implications in polycystic ovary syndrome. Eur J Endocrinol 2010;162:193-212.

7. Zhou G, Myers R, Li Y, Chen Y, Shen X, Fenyk-Melody J, et al. Role of AMP-activated protein kinase in mechanism of metformin action. J Clin Invest 2001;108:1167-74.

8. Gilbert MP, Pratley RE. The impact of diabetes and diabetes medications on bone health. Endocr Rev 2015;36:194-213.

9. Melton LJ, Leibson CL, Achenbach SJ, Therneau TM, Khosla S. Fracture risk in type 2 diabetes: update of a population-based study. J Bone Miner Res 2008;23:1334-42.

10. Delmas P, Eastell R, Garnero P, Seibel M, Stepan J. The use of biochemical markers of bone turnover in osteoporosis. Osteoporosis Int 2000;11:S2-17.

11. Vasikaran S, Eastell R, Bruyère $O$, Foldes A, Garnero $P$, Griesmacher A, et al. Markers of bone turnover for the prediction of fracture risk and monitoring of osteoporosis treatment: a need for international reference standards. Osteoporosis Int 2011:22:391-420.

12. Vasikaran S, Cooper C, Eastell R, Griesmacher A, Morris HA, Trenti T, et al. International Osteoporosis Foundation and International Federation of Clinical Chemistry and Laboratory Medicine position on bone marker standards in osteoporosis. Clin Chem Lab Med 2011;49:1271-4.

13. Morin-Papunen L, Rantala AS, Unkila-Kallio L, Tiitinen A, Hippeläinen M, Perheentupa A, et al. Metformin improves pregnancy and live-birth rates in women with polycystic ovary syndrome (PCOS): a multicenter, doubleblind, placebo-controlled randomized trial. J Clin Endocrinol Metab 2012; 97:1492-500.

14. Rotterdam ESHRE/ASRM-Sponsored PCOS consensus workshop group. Revised 2003 consensus on diagnostic criteria and long-term health risks related to polycystic ovary syndrome (PCOS). Hum Reprod 2004;19: 41-7.
15. Gennarelli G, Holte J, Berglund L, Berne C, Massobrio M, Lithell H. Prediction models for insulin resistance in the polycystic ovary syndrome. Hum Reprod 2000;15:2098-102.

16. Nestler JE, Jakubowicz DJ. Lean women with polycystic ovary syndrome respond to insulin reduction with decreases in ovarian $\mathrm{P} 450 \mathrm{c} 17 \alpha$ activity and serum androgens. J Clin Endocrinol Metab 1997;82:4075-9.

17. Morin-Papunen L, Vauhkonen I, Koivunen R, Ruokonen A, Martikainen H, Tapanainen JS. Metformin versus ethinyl estradiol-cyproterone acetate in the treatment of nonobese women with polycystic ovary syndrome: a randomized study. J Clin Endocrinol Metab 2003;88:148-56.

18. Morin-Papunen LC, Vauhkonen I, Koivunen RM, Ruokonen A, Martikainen HK, Tapanainen JS. Endocrine and metabolic effects of metformin versus ethinyl estradiol-cyproterone acetate in obese women with polycystic ovary syndrome: a randomized study. J Clin Endocrinol Metab 2000;85:3161-8.

19. Matthews DR, Hosker JP, Rudenski AS, Naylor BA, Treacher DF, Turner RC. Homeostasis model assessment: insulin resistance and beta-cell function from fasting plasma glucose and insulin concentrations in man. Diabetologia 1985;28:412-9.

20. Matsuda M, DeFronzo RA. Insulin sensitivity indices obtained from oral glucose tolerance testing: comparison with the euglycemic insulin clamp. Diabetes Care 1999;22:1462-70.

21. Srivastava AK, Vliet EL, Michael Lewiecki E, Maricic M, Abdelmalek A, Gluck $O$, et al. Clinical use of serum and urine bone markers in the management of osteoporosis. Curr Med Res Opin 2005;21:1015-26.

22. Ivaska KK, Lenora J, Gerdhem P, Akesson K, Väänänen HK, Obrant KJ. Serial assessment of serum bone metabolism markers identifies women with the highest rate of bone loss and osteoporosis risk. J Clin Endocrinol Metab 2008;93:2622-32.

23. Kanazawa I, Yamaguchi T, Yano S, Yamauchi M, Sugimoto T. Metformin enhances the differentiation and mineralization of osteoblastic MC3T3-E1 cells via AMP kinase activation as well as eNOS and BMP-2 expression. Biochem Biophys Res Commun 2008;375:414-9.

24. Molinuevo MS, Schurman L, McCarthy AD, Cortizo AM, Tolosa MJ, Gangoiti MV, et al. Effect of metformin on bone marrow progenitor cell differentiation: in vivo and in vitro studies. J Bone Miner Res 2010;25: 211-21.

25. Cortizo AM, Sedlinsky C, McCarthy AD, Blanco A, Schurman L. Osteogenic actions of the anti-diabetic drug metformin on osteoblasts in culture. Eur J Pharmacol 2006;536:38-46.

26. Kasai T, Bandow K, Suzuki H, Chiba N, Kakimoto K, Ohnishi T, et al. Osteoblast differentiation is functionally associated with decreased AMP kinase activity. J Cell Physiol 2009;221:740-9.

27. Wu W, Ye Z, Zhou Y, Tan W. AICAR, a small chemical molecule, primes osteogenic differentiation of adult mesenchymal stem cells. Int J Artif Organs 2011;34:1128-36.

28. Gao Y, Li Y, Xue J, Jia Y, Hu J. Effect of the anti-diabetic drug metformin on bone mass in ovariectomized rats. Eur J Pharmacol 2010;635: 231-6.

29. Mai Q, Zhang Z, Xu S, Lu M, Zhou R, Zhao L, et al. Metformin stimulates osteoprotegerin and reduces RANKL expression in osteoblasts and ovariectomized rats. J Cell Biochem 2011;112:2902-9.

30. Jeyabalan J, Viollet B, Smitham P, Ellis S, Zaman G, Bardin C, et al. The anti-diabetic drug metformin does not affect bone mass in vivo or fracture healing. Osteoporosis Int 2013;24:2659-70.

31. Monami M, Cresci B, Colombini A, Pala L, Balzi D, Gori F, et al. Bone fractures and hypoglycemic treatment in type 2 diabetic patients: a casecontrol study. Diabetes Care 2008;31:199-203.

32. Borges J, Bilezikian J, Jones-Leone A, Acusta A, Ambery P, Nino A, et al. A randomized, parallel group, double-blind, multicentre study comparing the efficacy and safety of Avandamet (rosiglitazone/metformin) and metformin on long-term glycaemic control and bone mineral density after 80 weeks of treatment in drug-naïve type 2 diabetes mellitus patients. Diabetes Obes Metab 2011;13:1036-46.

33. Glover SJ, Garnero P, Naylor K, Rogers A, Eastell R. Establishing a reference range for bone turnover markers in young, healthy women. Bone 2008;42: 623-30. 


\section{ORIGINAL ARTICLE: REPRODUCTIVE ENDOCRINOLOGY}

34. Reid IR. Relationships among body mass, its components, and bone. Bone 2002;31:547-55.

35. Hoppé E, Bouvard B, Royer M, Audran M, Legrand E. Sex hormone-binding globulin in osteoporosis. Joint Bone Spine 2010;77:306-12.

36. Ehrlich P, Lanyon L. Mechanical strain and bone cell function: a review. Osteoporosis Int 2002;13:688-700.

37. Lingaiah S, Morin-Papunen L, Piltonen T, Puurunen J, Sundström-Poromaa I, Stener-Victorin E, et al. Bone markers in polycystic ovary syndrome: a multicentre study. Clin Endocrinol (Oxf) 2017;87:673-9.

38. Kos E, Liszek M, Emanuele M, Durazo-Arvizu R, Camacho P. Effect of metformin therapy on vitamin D and vitamin B12 levels in patients with type 2 diabetes mellitus. Endocr Pract 2011;18:179-84.

39. Out M, Top WMC, Lehert P, Schalkwijk CA, Stehouwer CDA, Kooy A. Longterm treatment with metformin in type 2 diabetes and vitamin $D$ levels: a post-hoc analysis of a randomized placebo-controlled trial. Diabetes Obes Metab 2018;20:1951-6.

40. Alkharfy KM, Al-Daghri NM, Sabico SB, Al-Othman A, Moharram O, Alokail MS, et al. Vitamin D supplementation in patients with diabetes mellitus type 2 on different therapeutic regimens: a one-year prospective study. Cardiovasc Diabetol 2013;12:113.

41. Holick MF, Binkley NC, Bischoff-Ferrari HA, Gordon CM, Hanley DA, Heaney RP, et al. Evaluation, treatment, and prevention of vitamin D deficiency: an Endocrine Society clinical practice guideline. J Clin Endocrinol Metab 2011;96:1911-30.

42. Thomson RL, Spedding S, Buckley JD. Vitamin D in the aetiology and management of polycystic ovary syndrome. Clin Endocrinol (Oxf) 2012;77: 343-50.

43. Harada S, Rodan GA. Control of osteoblast function and regulation of bone mass. Nature 2003;423:349.

44. Parfitt AM. Osteonal and hemi-osteonal remodeling: the spatial and temporal framework for signal traffic in adult human bone. J Cell Biochem 1994;55:273-86.

45. Blumsohn A, Naylor KE, Timm W, Eagleton AC, Hannon RA, Eastell R. Absence of marked seasonal change in bone turnover: a longitudinal and multicenter cross-sectional study. J Bone Miner Res 2003;18: $1274-81$. 